\title{
Radio network planning and human exposure to electromagnetic fields
}

\author{
M. A. Baldauf, W. Sörgel, and W. Wiesbeck \\ Institut für Höchstfrequenztechnik und Elektronik (IHE), Universität Karlsruhe (TH), Germany
}

\begin{abstract}
This paper analyzes the effect of a change in cell size and of transmit power control on the human exposure to electromagnetic fields. In a simplified network configuration the well accepted COST-Hata model is considered to assess the path loss. It is shown that increasing the base station density will not generally raise the exposure but can rather help to lower it significantly. The exposure due to base stations is hardly affected by allowing a transmit power reduction at the base station for individual links. In contrast to this, it is demonstrated that transmit power control at the mobile station is a helpful means to lower the exposure efficiently.
\end{abstract}

\section{Introduction}

Information is of great value in everyday life. To spread information in a contemporary manner it should be sent at the speed of light via electromagnetic fields or waves either by wired or by wireless techniques. Concerning the wireless radio techniques information has to be transmitted via air resulting in a human exposure to electromagnetic fields. Popular limit values result in safety distances of just a few meters for typical base station configurations. Therefore, the human exposure to electromagnetic fields is typically not of primary interest in network planning since the resulting field strengths are well below the limit values, even in a close distance to the transmitters.

Base stations serve as air interfaces of the mobile radio network. Their equipment and configuration are chosen to provide mobile services to customers within a limited area. For capacity reasons more base stations are needed in areas with more customers. In this paper it is investigated whether an increase in the number of base stations will increase the human exposure to electromagnetic fields or not.

Every base station has to transmit cell specific information in a broadcast mode with a fixed transmit power. Transmit

Correspondence to: M. A. Baldauf

(michael.baldauf@ihe.uka.de) power control allows the transmission of information dedicated to individual users with a power level that is lower than a scheduled maximum transmit power. This results in a reduction of interference and, furthermore, in a reduction of the human exposure to electromagnetic fields. In this paper the amount of the decrease in the exposure to electromagnetic fields when utilizing transmit power control is quantified.

\section{The mobile radio network model}

To assess the human exposure to electromagnetic fields in a radio network, knowledge of the most important factors influencing the exposure is necessary. Aside from the network layout and wave propagation model, the base station's and mobile station's technical data and configuration are relevant.

\subsection{The network layout and propagation model}

A homogenous area is subdivided into hexagonal cells (cf. Fig. 1). Two rings of hexagons are added around a center hexagon to take the impact of adjacent cells into account. Base stations are placed at the centers of the hexagons. All cells are identical in terms of the number of active users and technical configurations of the terminals. The exposure is determined for the center cell. Simulations have shown that the increase in exposure due to adding two further rings around the center cell is only a few tenths of a $\mathrm{dB}$, and can thus be regarded as negligible.

In the middle of Fig. 1 the definition of the cell radius $R_{\mathrm{C}}$ and the orientation of the considered sector antennas are shown. On the right hand side the notation of the angles in a spherical coordinate system is given.

The COST-Hata model is chosen to compute the path loss $L$. This model gives the median value of the path loss in an urban area. In order to also regard for the smaller path loss in areas with lower building density a parameter $b$ describing the building development is taken into account. For open areas $b=0$ whereas in urban areas $b=1$, which holds for medium sized cities and suburban centers with medium tree 

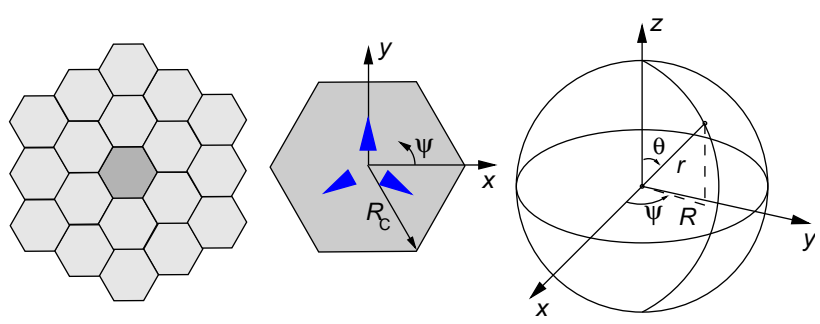

Fig. 1. Left: Network layout. A center hexagon is surrounded by two rings of hexagons. Middle: Definition of the cell radius $R_{\mathrm{C}}$ and orientation of the sector antennas (arrows). Right: Convention for angles in a spherical coordinate system.

density. By choosing $b$ to be a variable it is possible to assess the median path loss $L$ for arbitrary building developments $b$. As frequency $f=2 \mathrm{GHz}$ is considered. The exposure to electromagnetic fields is evaluated at a height of $1.5 \mathrm{~m}$ which corresponds to the height of the mobile station's antenna. Thus, the median value of the path loss $L$ based on the COST-Hata model is calculated here by:

$$
\begin{aligned}
\frac{L}{\mathrm{~dB}}= & 125.64-13.82 \log \left(\frac{h_{\mathrm{BS}}}{\mathrm{m}}\right)+32.52 b \\
& +\left(44.9-6.55 \log \left(\frac{h_{\mathrm{BS}}}{\mathrm{m}}\right)\right) \log \left(\frac{R}{\mathrm{~km}}\right)
\end{aligned}
$$

A standard deviation of $7 \mathrm{~dB}$ is considered in the simulations. Therefore, the average value of the path loss $L$ in a distance $R$ is $5.6 \mathrm{~dB}$ higher than the median value. No difference between uplink and downlink frequency is considered since the frequency difference is usually small in prevalent frequency division duplex (FDD) systems, and therefore has only a negligible influence on the path loss $L$.

The COST-Hata model is deemed to be valid in the frequency range of $1.5 \mathrm{GHz} \leq f \leq 2 \mathrm{GHz}$ for distances of $1 \mathrm{~km} \leq R \leq 20 \mathrm{~km}$, base station antenna heights of $30 \mathrm{~m} \leq h_{\mathrm{BS}} \leq 200 \mathrm{~m}$, and mobile station antenna heights of $1 \mathrm{~m} \leq h_{\mathrm{MS}} \leq 10 \mathrm{~m}$, respectively. In the calculation results presented here the COST-Hata model is applied down to base station antenna heights of $h_{\mathrm{BS}}=10 \mathrm{~m}$, which is valid as long as the base station antenna height $h_{\mathrm{BS}}$ is well above the buildings in the surroundings. Furthermore, the COST-Hata model is applied also for distances $R$ smaller than $1 \mathrm{~km}$. Path losses $L$ smaller than the free space value are replaced by the corresponding free space value.

\subsection{Base station}

The simulation results presented here assume a base station antenna height of $h_{\mathrm{BS}}=10 \mathrm{~m}$ for a cell radius of $R_{\mathrm{C}}=500 \mathrm{~m}$, increasing linearly to $h_{\mathrm{BS}}=60 \mathrm{~m}$ for a cell radius of $R_{\mathrm{C}}=10 \mathrm{~km}$. Regarding only macrocells, the antenna heights $h_{\mathrm{BS}}$ are assumed to always be above the height of buildings.
As base station antenna the Kathrein antenna type 742212 is chosen. This is a typical base station antenna with a gain of $G_{\mathrm{BS}} \approx 18 \mathrm{dBi}$. Its half power beamwidth is $\theta_{3 \mathrm{~dB}} \approx 7^{\circ}$ in elevation and $\psi_{3 \mathrm{~dB}} \approx 65^{\circ}$ in azimuth. The considered sector antennas are tilted down slightly. The downtilt angle is chosen in such a manner that the direction in which the antenna has its half power beamwidth above the main beam direction is directed at the height of a mobile station's antenna at the cell radius $R_{\mathrm{C}}$. Taking the dependence of the base station antenna height $h_{\mathrm{BS}}$ on the cell radius $R_{\mathrm{C}}$ into account, the antenna is declined electrically by some $4^{\circ}$. A three sector configuration with main beam directions of $\psi=\left\{90^{\circ}, 210^{\circ}, 330^{\circ}\right\}$ is chosen for Kathrein's 742212 (cf. Fig. 1).

Most calculations are performed for a system which is based on GSM (Global System for Mobile Communications). In GSM time division multiple access (TDMA) separates a carrier into eight time slots. Each active user in a cell occupies one time slot for transmission. There is a broadcast control channel occupying one time slot which needs to be continually transmitted for cell identification. The carrier containing this channel is the $\mathrm{BCCH}$ carrier. The remaining seven timeslots of the BCCH carrier can be filled with other information. If not all time slots are needed for users or signalling information, they are transmitted anyway containing dummy bursts. The simulations are performed under the assumptions that the mandatory $\mathrm{BCCH}$ carrier should be kept clear of user specific data and that all signalling is handled by this carrier.

The sensitivity level of the mobile station and the calculated minimum value of the path loss determine the power that is necessary to transmit the mandatory $\mathrm{BCCH}$ carrier. In addition to Eq. (1) a $3 \mathrm{~dB}$ loss due to the user's head is taken into account. A further $10 \mathrm{~dB}$ are added to the transmit power to assure also a reliable connection in the case of fading that cannot be overcome by GSM's quite slow transmit power control.

For time slots which are not transmitted on the $\mathrm{BCCH}$ carrier an optional transmit power control is possible (cf. Fig. 2). According to the specifications a maximum power reduction of $\Delta \mathrm{PC}_{\max }=30 \mathrm{~dB}$ compared to the transmit power of the $\mathrm{BCCH}$ carrier is allowed in time slots serving users at positions where the path loss permits a reduction of transmit powers. In Sect. 4.2.1 $\triangle \mathrm{PC}_{\max }$ is varied.

In these theoretical considerations the applied power control does not restrict the transmit power in a time slot to distinct stepped power levels, and the maximum transmit power is not limited. The regarded scenario is assumed to be in a steady state, i.e. specific power control procedures during and after the setting up of a connection are not regarded.

\subsection{Mobile station}

The mobile station's antenna height $h_{\mathrm{MS}}$ is chosen to be $1.5 \mathrm{~m}$ above the ground. An isotropic radiator $\left(G_{\mathrm{MS}}=0 \mathrm{dBi}\right)$ is considered as antenna. 
For GSM only speech transmissions are regarded with one time slot per active user. Again a $3 \mathrm{~dB}$ increase in transmit power due to the loss of the user's head and a further $10 \mathrm{~dB}$ to assure a reliable connection are taken into account. The average transmit power is one eighth of the power per time slot. Discontinuous transmission (DTX), which would lead to a lower average transmit power, is not considered.

For UMTS services a uplink data rate of $12.2 \mathrm{kbps}$ is considered. Except for the $3 \mathrm{~dB}$ increase in transmit power due to the loss of the user's head there is no additional increase in transmit power.

\subsection{User density/network load}

The mobile stations are assumed to be equally distributed over the cell area. When changing the cell size three cases of active user densities $n$ are considered:

- network without users, $n=0 / \mathrm{km}^{2}$

- very low active user density, $n=1 / \mathrm{km}^{2}$

- medium active user density, $n=100 / \mathrm{km}^{2}$

At hot spots higher user densities $n$ are possible, however, these higher user densities would demand smaller cell sizes than those regarded. For these pico- and microcellular cells the considered COST-Hata model is not applicable and a precise information about the propagation scenario would be indispensable.

When considering the effect of transmit power control onto the human exposure to electromagnetic fields the active user densities $n$ are chosen in order to transmit on one, four or six carriers $N$, which are reasonable numbers.

The user density is proportional to the load of the network. For this reason, a larger active user density corresponds to a higher load in the network.

\section{Exposure assessment}

Since the exposure is determined at least several meters away from the base station antenna, the reactive near field surrounding an antenna is not of interest. In the radiating near field a smoothing and limitation of the radiation pattern conservatively accounts for the not fully developed far field characteristic. Therefore, a simple far field calculation is done here to assess the exposure to electromagnetic fields.

Finding the total exposure $S_{\text {total }}$ at a certain height $z=h$ above the $(\mathrm{x}, \mathrm{y})$-plane can be achieved by summing all individual power densities due to each sector antenna. Since the individual field strengths produced by the base stations are uncorrelated the power densities can be simply added, yielding

$$
S_{\text {total }}(x, y, h)=\frac{4 \pi}{\lambda^{2}} \sum_{i} \frac{P_{\mathrm{T}, \mathrm{BS}} G_{\mathrm{BS}} C_{\mathrm{BS}, i}^{2}(\theta, \psi)}{L_{i}(x, y, h)} .
$$

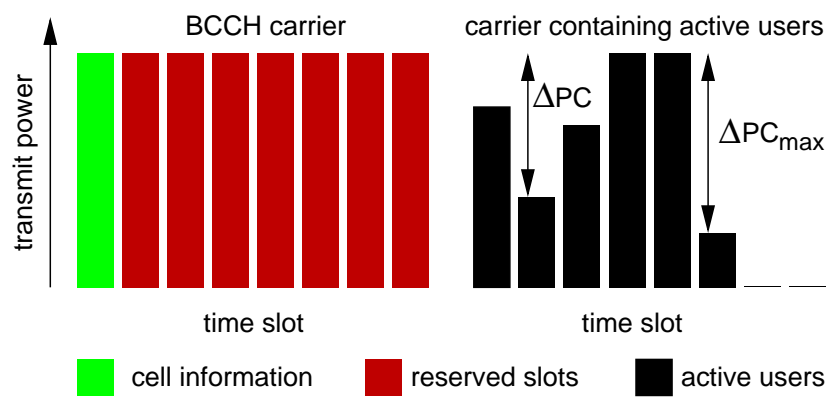

Fig. 2. Channel configuration of the considered GSM system. Power control is possible on carriers containing active users.

Herein $P_{\mathrm{T}, \mathrm{BS}}$ is the transmit power of the base station, $G_{\mathrm{BS}}$ its antenna gain, $C_{\mathrm{BS}, i}(\theta, \psi)$ the pattern of the base station antenna, $L_{i}$ the isotropic path loss at the regarded position $(x, y, h)$ to the base station $i$, and $\lambda$ the wavelength. The term $4 \pi / \lambda^{2}$ is the reciprocal effective aperture area of the mobile station's isotropic antenna.

\section{Results}

For the evaluation of the influence of a change in cell size on the exposure to electromagnetic fields, the power density at the height of the mobile station $h_{\mathrm{MS}}=1.5 \mathrm{~m}$ is calculated for the modelled center hexagon (cf. Fig. 1) at approximately 100000 equidistant points. Since the power density and, therefore, the exposure to electromagnetic fields is dependent on position the average value of the power density is evaluated to obtain a statistically significant measure of the exposure. All calculations were performed with Matlab.

In Sect. 4.1 the effect of the changing the cell size on the exposure to electromagnetic fields caused by the base stations is evaluated for a GSM system. The maximum power reduction $\Delta \mathrm{PC}_{\max }$ is set to $\Delta \mathrm{PC}_{\max }=30 \mathrm{~dB}$, as specified. In Sect. 4.2.1 the maximum power reduction $\triangle \mathrm{PC}_{\max }$ is a variable. Sections 4.2.2 and 4.2.3 consider the effect of transmit power control in GSM and UMTS (Universal Mobile Telecommunications System) networks, respectively.

\subsection{The effect of changing the cell size}

Figure 3 shows the average power densities $S_{\text {avg versus the }}$ cell radius $R_{\mathrm{C}}$ in a network without active users $(n=0)$. The average power densities $S_{\text {avg }}$ for larger building developments $b$ are slightly higher. Decreasing the cell radius $R_{\mathrm{C}}$ generally yields slightly smaller values of the average power density $S_{\text {avg }}$ for all building developments $b$. In the case of no users in the network there is an insignificant maximum in the average power density $S_{\text {avg }}$. The occurrence and the position of this maximum is dependent on the chosen base station configuration. 


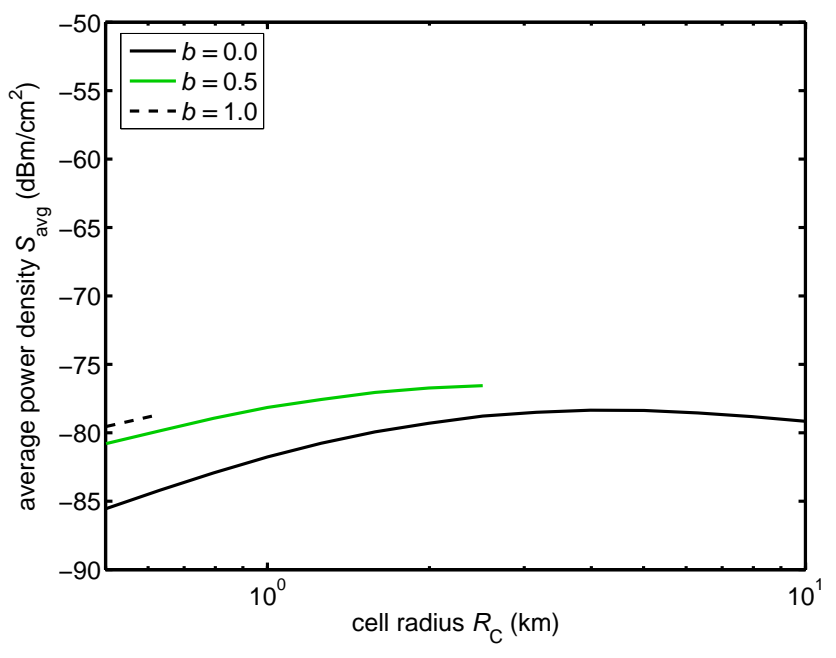

Fig. 3. Average value of the power density $S_{\text {avg }}$ versus the cell radius $R_{\mathrm{C}}$ for different building developments $b$. No active users in the network $(n=0)$.

The average power density $S_{\text {avg }}$ for cell radii $R_{\mathrm{C}}$ where a mobile station at the cell border cannot transmit the minimum required power level to the base station are not shown. Therefore, the lines for $b=0.5$ and $b=1.0$ end before reaching the maximum value of the abscissa.

Figure 4 illustrates that increasing the active user density $n$ and, therefore, the number of users in a cell - or the network load - will increase the average power density $S_{\text {avg }}$ since a higher transmit power per sector is needed to serve the customers with user specific information. The curves approach each other with decreasing cell radius $R_{\mathrm{C}}$. This is due to the dominant contribution of the mandatory $\mathrm{BCCH}$ carrier to the transmit power in smaller cells containing fewer active users. In networks containing active users there is a clear trend to a lower exposure to electromagnetic fields when decreasing the cell size. The same characteristics can be found for the median value and the 95 th percentile of the power density.

Since the resources are limited, the case of 100 active users per square kilometer typically cannot be realized with a cell radius of $R_{\mathrm{C}}=10 \mathrm{~km}$. Depending on the number of available frequencies and their re-use factor, the cell radius $R_{\mathrm{C}}$ might be smaller, e.g. a few kilometers.

Cooper et al. (2004) report that measurements show a higher exposure to electromagnetic fields close to the base station antennas of microcells than of macrocells. Coopers' results may appear to conflict with those presented here, but the different base station and cell parameters used here explain the seeming disparity. The antennas of microcellular base stations investigated by Cooper et al. (2004) are mounted at heights of $2.5 \mathrm{~m} \leq h_{\mathrm{BS}} \leq 9 \mathrm{~m}$ and show broader elevation patterns than the antennas mounted at macrocellular sites. In this study only macrocells with base station antenna heights of $h_{\mathrm{BS}} \geq 10 \mathrm{~m}$ are regarded. Moreover, the antenna

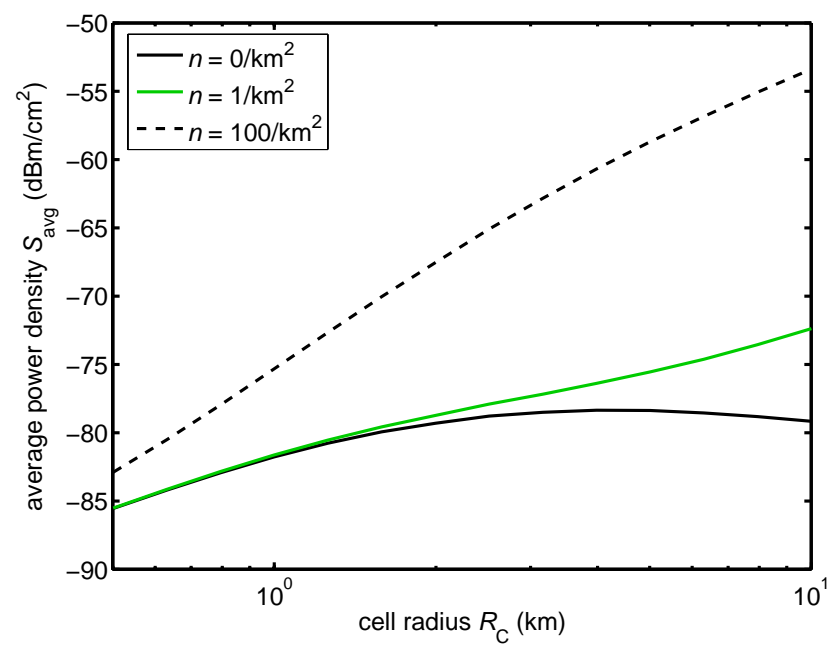

Fig. 4. Average value of the power density $S_{\text {avg }}$ versus the cell radius $R_{\mathrm{C}}$ for different numbers of active user densities $n$. $b=0$.

type considered here is independent of the cell size. Solely the electrical downtilt is increased by a small amount when decreasing the cell radius.

\subsection{The effect of transmit power control}

Transmit power control is a means to transmit information which needs not to be received by everybody in the cell with a output power that is lower than a scheduled maximum value. Emitting a lower power might help to decrease the interference if the same resources are used in nearby cells. In well planned GSM systems and UMTS downlinks interference is not significant. However, interference is of great impact on the performance of a system in the case of the uplink in CDMA (code division multiple access) systems. In addition, transmit power control is an efficient feature to increase the battery lifetime of mobile stations.

For mobile stations the exposure to electromagnetic fields in the human head is of primary interest. Since the exposure scenario consists of an inhomogeneous head in the near field of a source the specific absorption rate (SAR) needs to be determined when the absolute value of the exposure is of interest. For a relative comparison of the exposure to electromagnetic fields of mobile stations, it is sufficient to compare the relative changes in the transmit powers $P_{\mathrm{T}, \mathrm{MS}}$ of the mobile stations. This is due to the fact that the path loss between the mobile terminal and the absorbing human does not change when changing the cell size.

\subsubsection{Transmit power control at the base station}

Figure 5 shows the effect of transmit power control on the exposure to electromagnetic fields for a fixed cell size $R_{\mathrm{C}}=1 \mathrm{~km}$ and medium building development $b$. The active user densities $n$ are chosen in order to allocate one, four 


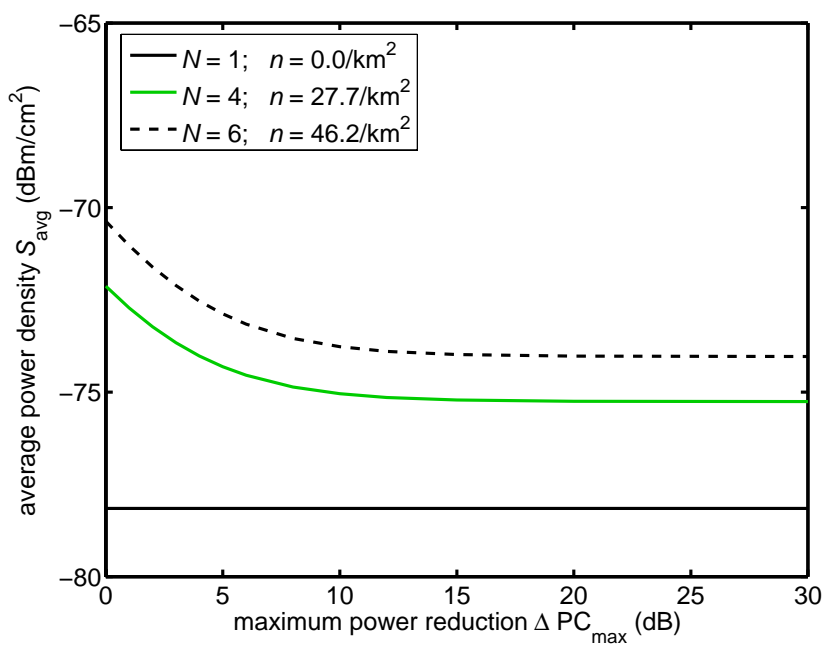

Fig. 5. Average power density $S_{\text {avg }}$ versus the maximum power reduction $\Delta \mathrm{PC}_{\max } \cdot R_{\mathrm{C}}=1 \mathrm{~km}, b=0.5$.

and six carriers $N$ per sector. In the case of just one carrier the human exposure to electromagnetic fields is independent of the maximum power reduction $\triangle \mathrm{PC}_{\max }$ since the mandatory $\mathrm{BCCH}$ carrier is not power controlled. Transmitting on four carriers with full power will increase the exposure by $6 \mathrm{~dB}$ if the maximum power reduction $\triangle \mathrm{PC}_{\max }=0$. By increasing the value of $\Delta \mathrm{PC}_{\max }$ the average power density $S_{\mathrm{avg}}$ decreases since part of the active users are well served even with a smaller transmit power of the link. Assuming equally distributed users a maximum power reduction $\triangle \mathrm{PC}_{\max }$ of more than $10 \mathrm{~dB}$ will not allow a further significant reduction of the exposure. For example in the case of four carriers a figure of $\triangle \mathrm{PC}_{\max }=10 \mathrm{~dB}$ will allow a power reduction of $2.9 \mathrm{~dB}$, and $\Delta \mathrm{PC}_{\max }=30 \mathrm{~dB}$ a power reduction of $3.4 \mathrm{~dB}$, respectively. Subtracting the uncontrolled BCCH carrier shows that the transmit power can be reduced by $5 \mathrm{~dB}$ for the power controlled channels. However, due to the mandatory $\mathrm{BCCH}$ carrier the exposure reduction is lower. For other cell radii a similar exposure reduction can be achieved.

Simulations with a static network simulator for UMTS have shown that in networks which are based on CDMA the exposure reduction due to the base stations when decreasing the cell size or allowing a power control for user specific information is similar to that shown for GSM. In contrast to this, significant changes can be observed for the probability density functions of the mobile station's transmit powers (cf. Sect. 4.2.3).

\subsubsection{Transmit power control at the mobile station (GSM)}

The power control range of the mobile station allows the transmit power to be adjusted within the range of $0 \mathrm{dBm} \leq P_{\mathrm{T}, \mathrm{MS}} \leq 30 \mathrm{dBm}$. Figure 6 shows the probability density functions of the transmit powers $P_{\mathrm{T}, \mathrm{MS}}$ of the mobile terminals in a GSM network for two different cell sizes.

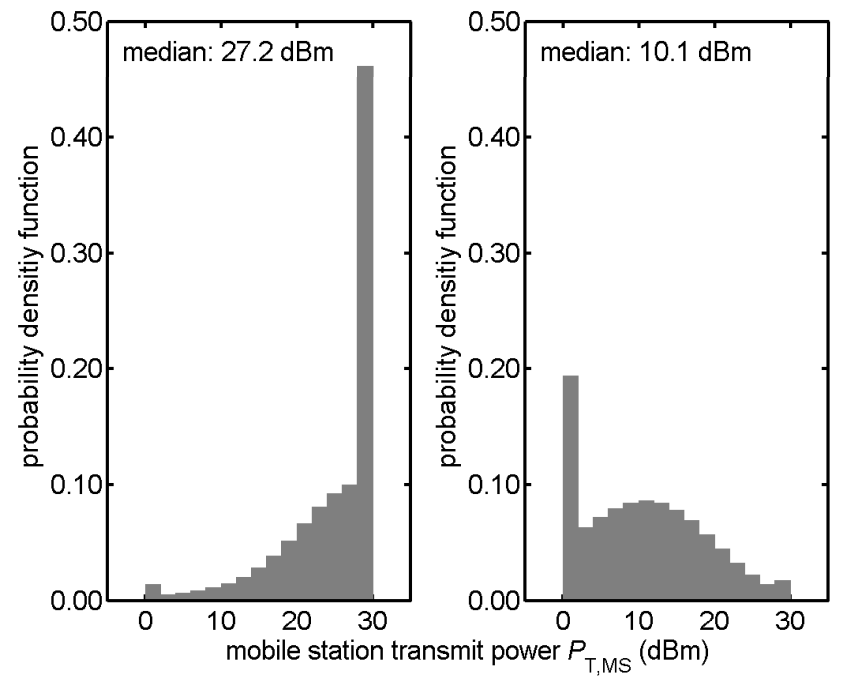

Fig. 6. Probability density functions of the transmit power of GSM mobile stations $P_{\mathrm{T}, \mathrm{MS}}$ in a steady state. Bin width: $2 \mathrm{~dB}$. Parameters: $b=0.2$; left hand side: $R_{\mathrm{C}}=6.3 \mathrm{~km}$; right hand side: $R_{\mathrm{C}}=1 \mathrm{~km}$.

The probability density function gives the probability of finding the transmit power in a certain range. The bin width was chosen to be $2 \mathrm{~dB}$, which corresponds to the transmit power control steps implemented in GSM. Without transmit power control all mobile stations would transmit with a power $P_{\mathrm{T}, \mathrm{MS}}=30 \mathrm{dBm}$.

On the left hand side of Fig. 6 the cell radius is $R_{\mathrm{C}}=6.3 \mathrm{~km}$, on the right hand side $R_{\mathrm{C}}=1 \mathrm{~km}$. The larger cell size demands a higher transmit power $P_{\mathrm{T}, \mathrm{MS}}$ from the large number of the mobile stations which are not in the range of the smaller cell. The median values of the transmit powers differ by some $17 \mathrm{~dB}$. Smaller cell sizes will in general lead to lower exposures due to the mobile terminals.

As can be seen from Fig. 6 a lot of mobiles are at the limit of either their maximum or minimum transmit power. The reason for this is that the path loss varies over a much larger range than the GSM power control range is specified for.

Micro- or picocellular base stations, not regarded here, might have a lower sensitivity. Therefore, the transmit powers of the mobile terminals in such cells might be higher than in a similar network where the base stations fulfill the specifications of macrocellular base stations.

\subsubsection{Transmit power control at the mobile station (UMTS)}

Figure 7 shows the probability density functions of the transmit powers $P_{\mathrm{T}, \mathrm{MS}}$ of UMTS mobile stations. The specifications demand that the transmit power can be decreased to at least $-50 \mathrm{dBm}$. Depending on the power class to which the mobile station belongs, the maximum transmit power ranges from $21 \mathrm{dBm}$ (class 4 ) to $33 \mathrm{dBm}$ (class 1 ). The much larger power control range allows a broader distribution of 


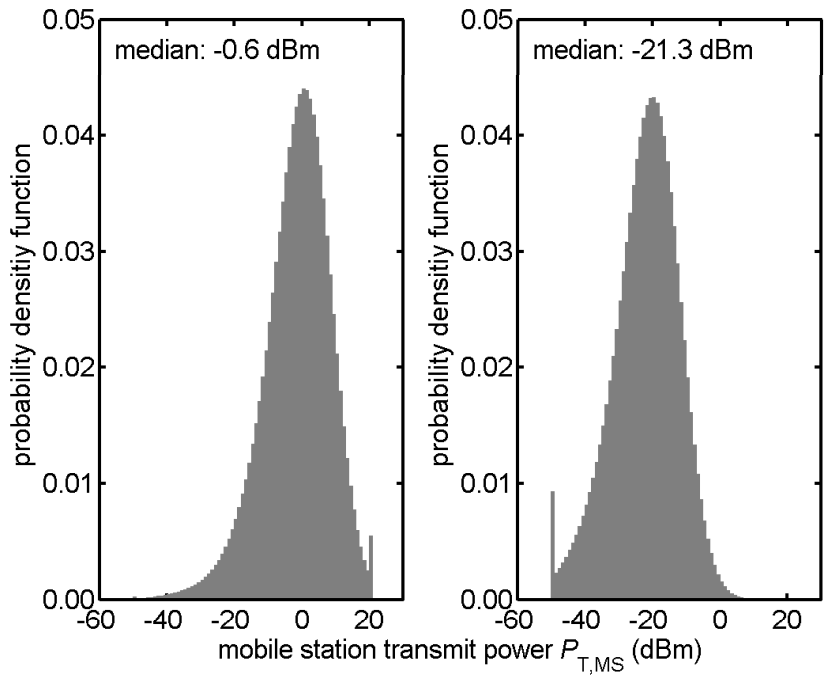

Fig. 7. Probability density functions of the transmit power of UMTS mobile stations $P_{\mathrm{T}, \mathrm{MS}}$ in a steady state. Bin width: $1 \mathrm{~dB}$. Parameters: $b=0.2$; uplink data rate: $12.2 \mathrm{kbps}$; channel model: vehicular A; mobile station speed: $3 \mathrm{~km} / \mathrm{h}$; left hand side: $R_{\mathrm{C}}=6.3 \mathrm{~km}$; right hand side: $R_{\mathrm{C}}=1 \mathrm{~km}$.

the probability density functions of the transmit power. For the smaller cell radius (right hand side of Fig. 7) some mobile stations would even profit from a lower minimum transmit power. UMTS will not work properly without transmit power control.

The median values of the transmit powers in both network configurations are much smaller than those in a GSM network. Aside from the fact that the transmit powers shown in Fig. 6 are not time averaged, which would result in a power reduction of some $9 \mathrm{~dB}$, interference plays a crucial role in CDMA networks. Any unneeded increase in transmit power would lead to an unwanted increase in the interference level at the base station. The very fast transmit power control of UMTS mobile stations allows a rapid adoption of the power level to changing propagation conditions. In contrast to this the transmit power of GSM systems might be adjusted very slowly. Due to the separation of the users in frequency and time domain interference is not a severe problem. Therefore, the transmit powers are increased well above the required minimum value in GSM systems.

\section{Conclusions}

The exposure to electromagnetic fields in a simplified mobile cellular network has been evaluated. It was shown that decreasing the cell size can help to lower the resulting power density. This is mainly due to the reduction of the network load. In areas where the load is low the installation of more base stations will not change the exposure to electromagnetic fields significantly. Therefore, in terms of exposure, there is no reason to limit the number of base stations. Transmit power control at the base stations results in a moderate reduction of the exposure to electromagnetic fields. However, mobile stations will benefit from transmit power control in combination with small cell sizes due to their intelligent power control algorithms. Radio network planning that keeps the request of low transmit powers at the mobile station in mind is an effective means to achieve a significant reduction of the human exposure to electromagnetic fields.

\section{References}

Balanis, C. A.: Antenna theory: A review, Proceedings of the IEEE, vol. 80, pp. 7-23, Jan., 1992.

Baldauf, M. A., Herschlein, A., Sörgel, W., and Wiesbeck, W.: Safety distances in mobile communications, in: Proceedings of 2nd International Workshop on Biological Effects of Electromagnetic Fields, Rhodes, Greece, pp. 148-156, Oct., 2002.

Baldauf, M. A., Knörzer, S., Sörgel, W., and Wiesbeck, W.: Cell splitting and exposure in GSM networks, in: 13th International Conference on Telecommunications, Funchal, Madeira, CDROM, May, 2006a.

Baldauf, M. A., Knörzer, S., Kayser, T., and Wiesbeck, W.: Transmit power control in mobile communications and its potential for a reduction of EMF exposure, in: Proceedings of 4th International Workshop on Biological Effects of Electromagnetic Fields, Crete, Greece, vol. 1, pp. 231-239, Oct., 2006b.

Cooper, T. G., Mann, S. M., Khalid, M., and Blackwell, R. P.: Exposure of the General Public to Radio Waves near Microcell and Picocell Base Stations for Mobile Telecommunications, HPA Centre for Radiation, Chemical and Environmental Hazards (Radiation Protection Division), online available: http://www.hpa.org.uk/radiation/publications/w_ series_reports/2004/nrpb_w62.pdf, Sept., 2004.

COST 231: Digital mobile radio towards future generation systems, European Commission, Directorate General XIII, EUR 18957, Tech. Rep., 1999.

ICNIRP: Guidelines for limiting exposure to time-varying electric, magnetic, and electromagnetic fields (up to $300 \mathrm{GHz}$ ), Health Physics, 74(4), 494-522, 1998.

IEEE Standard for Safety Levels with Respect to Human Exposure to Radio Frequency Electromagnetic Fields, $3 \mathrm{kHz}$ to $300 \mathrm{GHz}$, IEEE Std. C95.1-2005, 2006.

KATHREIN-Werke KG: Online available: http://www.kathrein. com/.

3rd Generation Partnership Project; Technical Specification Group GSM/EDGE Radio Access Network, Radio transmission and reception (Release 1999), Technical Specification, 3GPP TS 05.05, 2003.

3rd Generation Partnership Project; Technical Specification Group Radio Access Networks, User equipment (UE) radio transmission and reception (FDD) (Release 4), Technical Specification, 3GPP TS 25.101, 2004. 